\title{
Osteoma frontoetmoidal, otras aplicaciones del Draf III, a raíz de un caso clínico
}

\section{Frontoethmoidal osteoma, other applications of Draf III on the grounds of a clinical case}

\author{
Ximena Maul F1, Claudia González G², Claudio Callejas C².
}

\begin{abstract}
RESUMEN
Presentamos el caso de una mujer de 57 años, que consulta tras notar un aumento de volumen pétreo y de lento crecimiento en el reborde orbitario medial derecho, asociado a obstrucción nasal, anosmia y descarga posterior. La evaluación clínico radiológica evidenció un gran osteoma frontoetmoidal derecho con compromiso orbitario y de la lamela lateral de la lámina cribosa, y además, una rinosinusitis crónica con pólipos. Se realizó una resección endoscópica del tumor por medio de un Draf III y una cirugía endoscópica funcional del resto de las cavidades perinasales para el tratamiento de la rinosinusitis crónica. Describimos el caso y discutimos sus aspectos relevantes.
\end{abstract}

Palabras clave: Osteoma frontoetmoidal, Draf III.

\begin{abstract}
We report the case of a 57 year-old woman consulting because of a slow growing solid tumor in the right medial orbital rim associated with nasal obstruction, anosmia and posterior nasal drip. Clinical and radiological evaluation evidenced a large right frontoethmoidal osteoma involving the orbit and thelateral lamella of the cribriform plate, and chronic rhinosinusitis with polyps. A Draf III was performed to remove the osteoma and a bilateral functional endoscopic sinus surgery of the remaining sinuses was performed to treat thechronic rhinosinusitis. We described the case and discuss its relevant aspects.
\end{abstract}

Key words: Frontoethmoidal osteoma, Draf III.

1 Residente. Departamento Otorrinolaringología, Pontificia Universidad Católica de Chile.

2 Otorrinolaringólogo. Departamento Otorrinolaringología, Pontificia Universidad Católica de Chile.

Recibido el 4 de marzo de 2015. Aceptado el 2 de julio de 2015. 


\section{INTRODUCCIÓN}

Hace ya más de dos décadas que resurgió el procedimiento inicialmentedescrito porLothrop para el rescate del seno frontal por vía externa, para su ejecución en forma endonasal. La técnica endonasal inicialmente fue descrita bajo microscopía (Draf III) y luego en forma endoscópica (procedimiento de Lothrop endoscópico modificado) $)^{1,2}$. Esta última ha adquirido gran popularidad debido al importante desarrollo de la cirugía endoscópica nasosinusal y hoy ambos términos se usan indistintamente.

La técnica endonasal surgió como una alternativa a la desfuncionalización del seno frontal por medio de un flap osteoplástico (FOP) con obliteración grasa, considerado el estándar de oro por mucho tiempo para el manejo del seno frontal con un receso obliterado por tejido cicatricial.

El procedimiento consiste en crear la mayor comunicación posible entre ambos senos frontales y las fosas nasales. Para esto es necesario remover el piso de ambos senos frontales, el tabique interseno frontal y una porción del tabique nasal bajo Ios senos frontales para crear una ventana septal que permita trabajar desde una fosa nasal hacia la otra. Esto implica fresar el proceso frontal de los huesos maxilares y la espina nasal del frontal. Los límites de la disección a cada lado son: 1) límite anterior y lateral: el periostio bajo la piel de la región del nasion, 2) límite posterior: primera neurona olfatoria, que marca el inicio de la fosa craneal anterior (Figura 1$)^{2}$.

Con el tiempo se han incorporado otras aplicaciones al Draf III, como por ejemplo, la resección de tumores del seno frontal3,4. A continuación presentamos la reseción de un osteoma frontoetmoidal por medio de esta técnica.

\section{CASO CLÍNICO}

Este reporte de caso cuenta con la aprobación del Comité Ético Científico de la Facultad de Medicina de la Pontificia Universidad Católica de Chile.

Una mujer de 57 años fue evaluada en el Departamento de Otorrinolaringología de la Pontificia Universidad Católica de Chile en mayo del año 2013. Consultó por un aumento de volumen en el reborde orbitario medial derecho (ángulo del ojo), indoloro, de consistencia pétrea y lento crecimiento, el cual no producía diplopia. Además refería anosmia y al interrogatorio dirigido presentaba obstrucción nasal (con una magnitud de 3 en una escala de 0 a 5) y descarga posterior (con una magnitud de 3 en una escala de 0 a 5).

La evaluación endoscópica y por tomografía computarizada (TC) evidenció un extenso osteoma de la región frontoetmoidal derecha que comprometía la órbita, en estrecha relación con la tróclea del músculo oblicuo superior, y la base de cráneo, en íntima relación con la lamela lateral de la lámina cribosa y la arteria etmoidal anterior (Figura 2). Además se detectó la coexistencia de una rinosinusitis crónica con pólipos bilateral (Figura 3).

Se realizó una resección endoscópica del osteoma por medio de un Draf III y una cirugía endoscópica funcional bilateral de todas las cavidades perinasales restantes. La cirugía fue

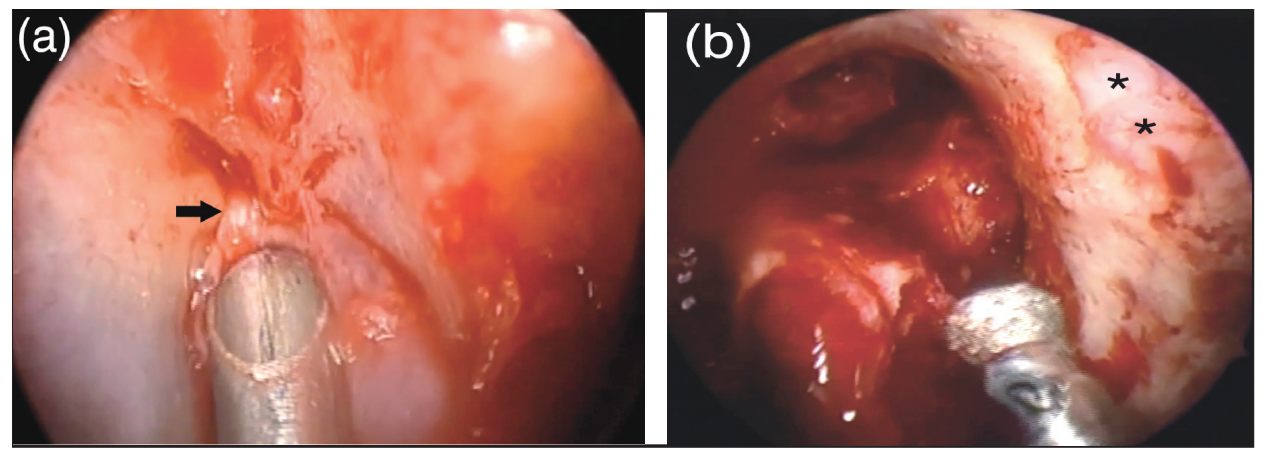

Figura 1. Límites de la disección del Draf III (imágenes tomadas en el intraoperatorio del caso). a) Se observa la primera neurona olfatoria izquierda (flecha), límite posterior de la disección en el Draf III. b) Se observa piel (asteriscos) en el límite anterolateral izquierdo del fresado. 


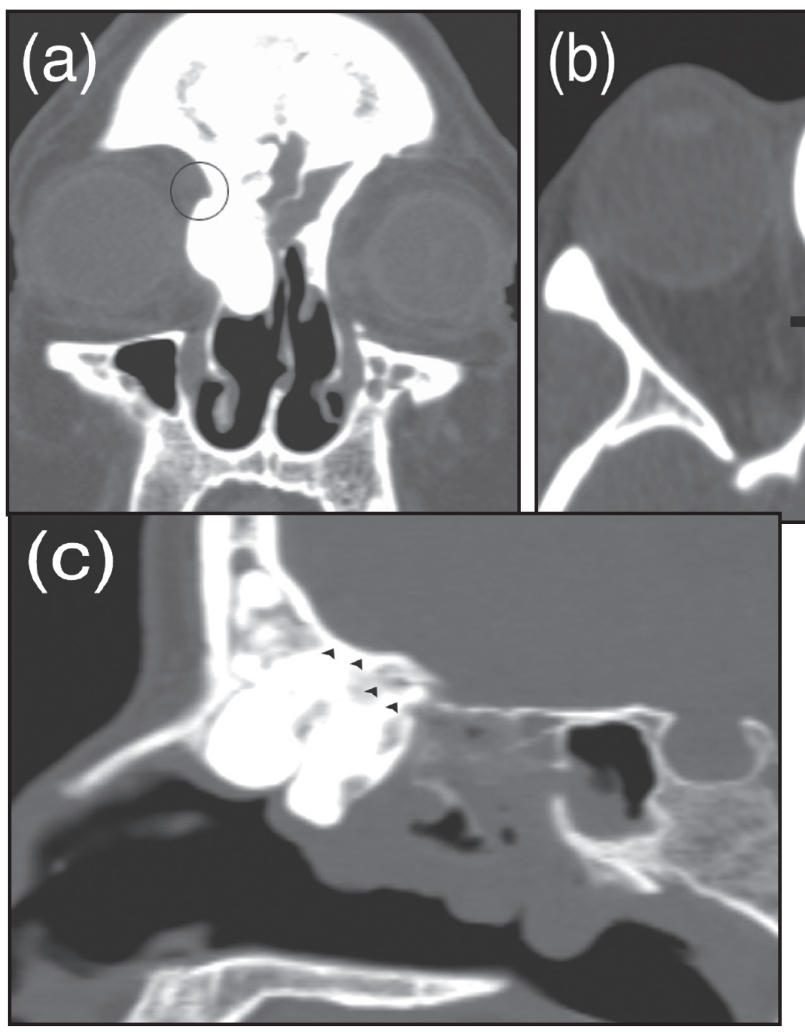

Figura 2. Escáner preoperatorio. A: corte coronal, el área al interior del círculo indica la zona de estrecha relación entre el componente orbitario del osteoma y la tróclea del músculo oblicuo superior derecho. B: corte axial, se observa la inserción del osteoma en la lamela lateral de la lámina cribosa (cabezas de flecha) y su íntima relación con la arteria etmoidal anterior (flecha). C: corte sagital, se aprecia la inserción del osteoma en la base de cráneo posterior al ostium del seno frontal.

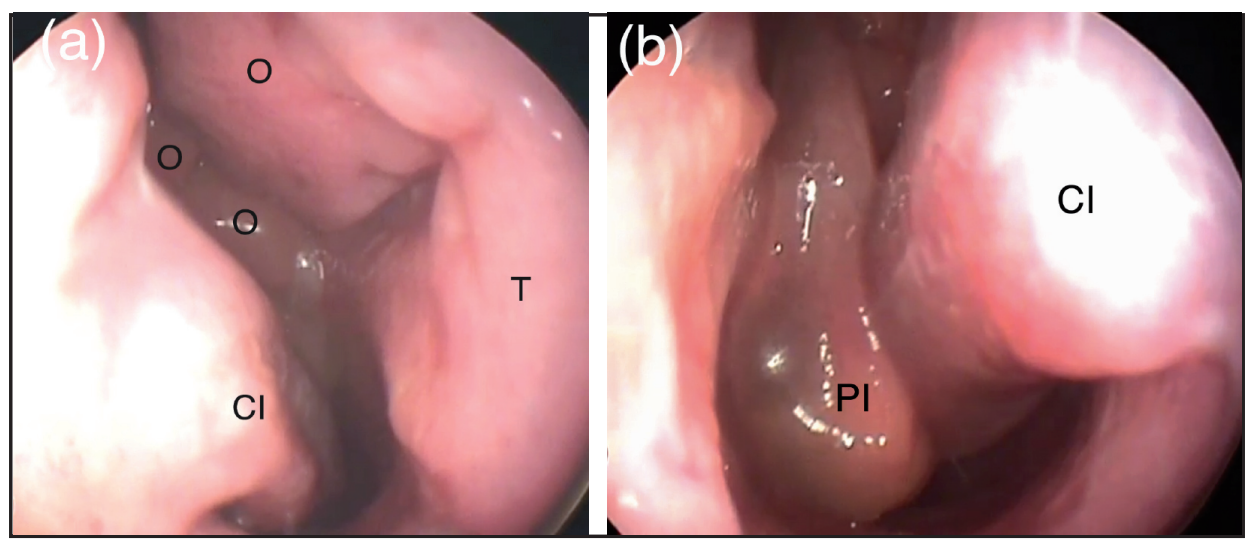

Figura 3. Endoscopía preoperatoria. a) Fosa nasal derecha. b) Fosa nasal izquierda. 0, osteoma; PI, pólipo inflamatorio; $\mathrm{Cl}$, cornete inferior; T, tabique nasal.

asistida por neuronavegador y tuvo una duración de 8 horas. En las zonas críticas de la disección el osteoma se fresó desde el centro hacia afuera con el fin de preservar la "cáscara" que conforma los bordes externos del tumor. Esto permitió fracturar cuidadosamente esta "cásca- ra” del componente orbitario, desde el ángulo interno del ojo en forma externa, y así lograr su resección completa sin lesionar la tróclea (Figura 4). La "cáscara" del osteoma se preservó en la región de la base de cráneo para evitar crear una fístula de líquido cerebroespinal o dañar la arteria 


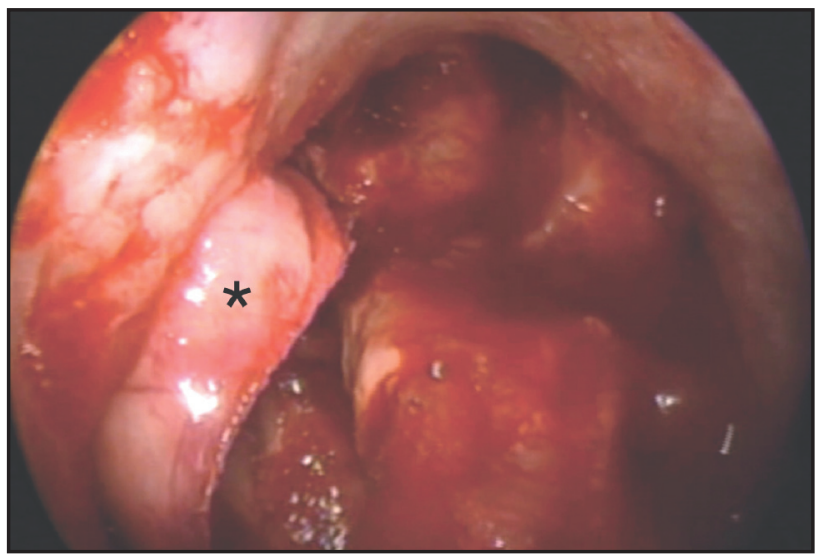

Figura 4. Se observa el momento en que se fractura el componente orbitario por vía externa logrando desplazarlo íntegro hacia la cavidad nasal (asterisco).

etmoidal anterior. La biopsia diferida corroboró el diagnóstico de osteoma cortical y de pólipos inflamatorios.

Tras finalizar la cirugía la paciente se trasladó a recuperación normal y posteriormente a una sala de complejidad estándar, sin taponamiento nasal. Evolucionó satisfactoriamente, sin diplopia, sin signos de fístula de líquido cerebroespinal ni otras complicaciones por lo que fue dada de alta al día siguiente de la operación. Al alta se indicaron lavados nasales de alto volumen, corticoides intranasales (mometasona, 2 puff en cada fosa nasal cada $12 \mathrm{hrs}$ ), curso de 3 semanas de antibiótico (doxiciclina $100 \mathrm{mg} \mathrm{c} / 12 \mathrm{hrs}$ ) y una dosis decreciente de prednisona. Asistió a controles periódicos para realizar curaciones.

En cuanto a sus síntomas, la paciente evolucionó satisfactoriamente refiriendo una resolución completa de la obstrucción nasal, descarga posterior e hiposmia, que se mantuvo hasta el último control vigente (a los 8 meses de la cirugía). En cuanto a la rinorrea fue evaluada con nota 1 en una escala de 0 a 5 puntos. Nunca presentó diplopia ni evidencias de fístula de líquido cerebroespinal y se planea un seguimiento a largo plazo para detectar un eventual crecimiento del remanente en base de cráneo y para manejo de su rinosinusitis crónica. Las Figuras 5 y 6 muestran la evolución posoperatoria de la paciente.

\section{DISCUSIÓN}

El procedimiento Draf III es una alternativa efectiva en la resección de tumores benignos del seno frontal; es una técnica segura, eficaz, bien tolerada por el paciente y tiene menos morbilidad que la vía abierta ${ }^{5}$. Además, tiene la ventaja de ser una cirugía de rápida recuperación en el posoperatorio, con posibilidad de alta hospitalaria incluso el mismo día de la cirugía. La gran abertura que se logra finalmente permite un adecuado control endoscópico de los senos frontales a largo plazo. Es un procedimiento demandante y requiere considerable entrenamiento en cirugía endoscópica ${ }^{5}$.

El abordaje del seno frontal por vía endoscópica (Draf III) no reemplaza al abordaje abierto (flap osteoplástico); dependiendo de las características del caso una vía será más conveniente que la otra. Si bien, en términos generales, el Draf III presenta menos morbilidad asociada, existen casos en que es mejor abordar el seno frontal por vía externa debido a la dificultad para la instrumentalización adecuada que significaría abordarlo en forma endoscópica ${ }^{6}$. Por ejemplo, cuando se requiere resecar lesiones en el límite superior, lateral 0 anterior de senos frontales amplios con recesos frontales de diámetro anteroposterior (relativamente estrecho) puede ser extremadamente difícil o imposible alcanzar la lesión en forma endoscópica. En estos casos la vía externa ofrece mayor comodidad para la instrumentalización al interior del seno. Además, 


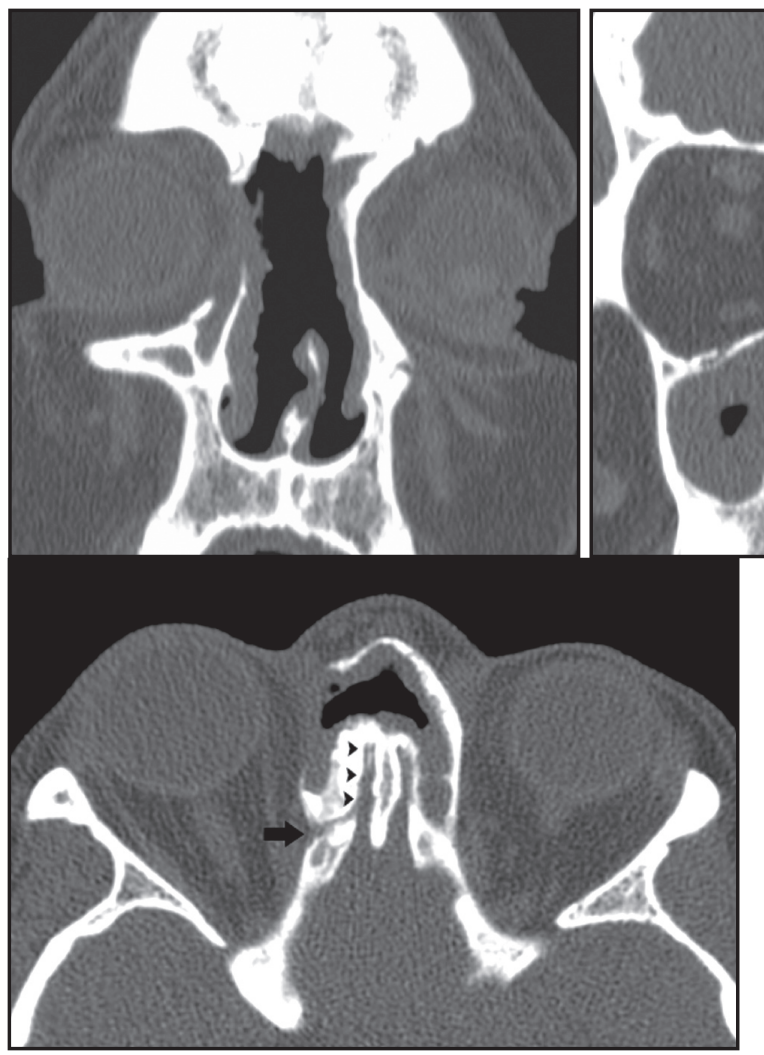

Figura 5. Escáner posoperatorio (3 meses). Se aprecia que la mayor parte del osteoma fue removido, dejando una pequeña "cáscara" adherida a la lamela lateral de la lámina cribosa en la base de cráneo para evitar una fístula de líquido cerebroespinal y dañar la arteria etmoidal anterior. El componente orbitario del osteoma se removió completamente, manteniendo indemnidad de las estructuras de la órbita. Los velamientos que se observan se deben a que la paciente cursaba una sobreinfección al momento del escáner. Cabezas de flecha, área de inserción de osteoma en lamela lateral de lámina cribosa; flecha, arteria etmoidal anterior.
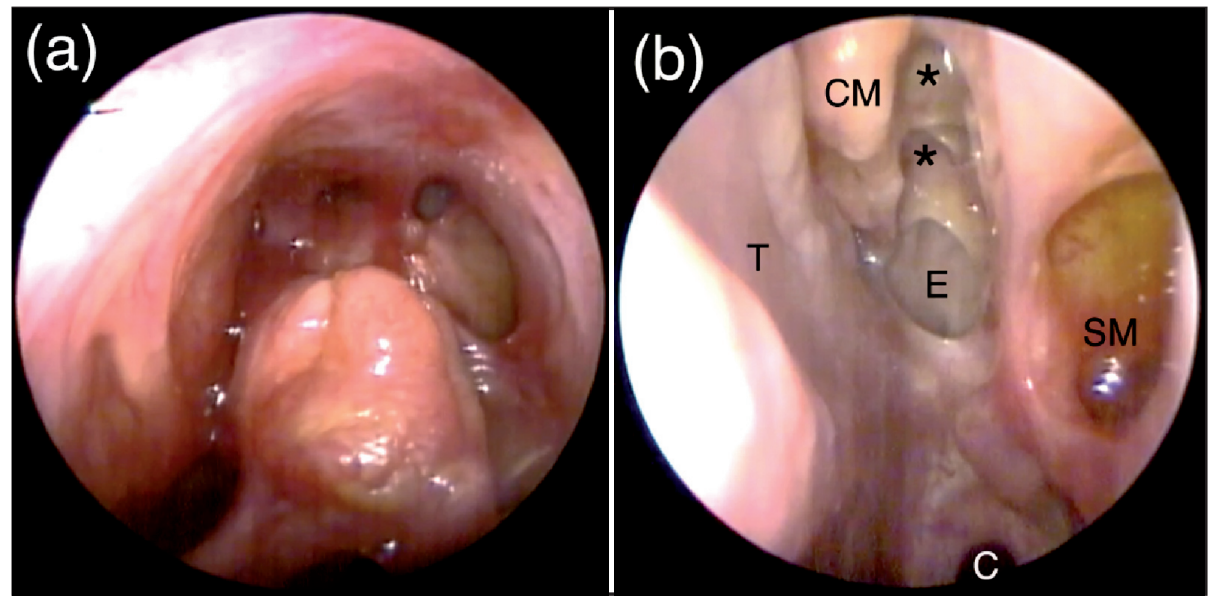

Figura 6. Endoscopía a los 8 meses de la cirugía. a) Se observa la región del Draf III amplia y cubierta con mucosa de aspecto sano. Se aprecia como con un endoscopio de $45^{\circ}$ se logran visualizar adecuadamente ambos senos frontales, incluso su pared anterior, lo que permite un adecuado seguimiento endoscópico a largo plazo. b) Se observa la fosa nasal izquierda libre de pólipos, con mucosa de aspecto sano.

T, tabique nasal; SM, seno maxilar; E, seno esfenoidal; asteriscos, base de cráneo; C, coana; CM, cornete medio parcialmente resecado. 
debido a lo laborioso de la resección endoscópica, muchos prefieren el abordaje externo para extraer osteomas extensos del seno frontal. En nuestro caso elegimos la vía endoscópica por las siguientes razones: 1) seno frontal pequeño con diámetro anteroposterior amplio, 2) gran extensión de la lesión hacia etmoides y órbita y 3) zona de implantación en base de cráneo anterior, posterior al "ostium" frontal.

Consideramos que el caso clínico presentaba 2 pasos críticos debido al gran riesgo de complicación, éstos son: 1) la resección del componente intraorbitario de la lesión, en estrecha relación con tróclea del músculo oblicuo superior, y 2) el fresado del área de implantación en la base de cráneo, en íntima relación con la lamela lateral de la lámina cribosa y la arteria etmoidal anterior. Para evitar lesionar la tróclea al resecar el componente intraorbitario de la lesión, se fresó el interior de éste dejando una delgada "cáscara ósea del osteoma" que fue luego fracturada hacia la fosa nasal desde el exterior, haciendo presión digital desde el ángulo interno del ojo derecho. Esto permitió retirar completamente el componente orbitario sin dañar la tróclea. En relación al sitio de implantación del osteoma en la base de cráneo, se decidió fresar y adelgazar este componente, pero no retirarlo en su totalidad, para así evitar el riesgo de fístula de líquido cerebroespinal y daño a la arteria etmoidal anterior

Si bien inicialmente el Draf III fue descrito para el manejo del seno frontal cicatricial en pacientes con rinosinusitis crónica sometidos a cirugías de revisión, con el desarrollo de la cirugía endoscópi- ca rápidamente se incorporaron otras aplicaciones. Entre éstas destacan: 1) la resección de tumores del seno frontal, como por ejemplo el papiloma schneideriano, 2) los abordajes de base de cráneo extendidos, específicamente como parte del abordaje transcribiforme, y 3) para el cierre de fístulas de líquido cerebroespinal originadas en la tabla posterior del seno frontal.

\section{BIBLIOGRAFÍA}

1. Draf W. Endonasal micro-endoscopic frontal sinus surgery: The fulda concept. Operative Techniques in Otolaryngology-Head and Neck Surgery 1991; 2: 234-40.

2. Wormald PJ. Salvage frontal sinus surgery: the endoscopic modified Lothrop procedure. Laryngoscope 2003; 113: 276-83.

3. Seiberling K, Floreani S, Robinson S, Wormald PJ. Endoscopic management of frontal sinus osteomas revisited. Am J Rhinol Allergy 2009; 23: 331-6.

4. Jiun Fong Thong, Deyal Chatterjee, Siew Yoong Hwang. Endoscopic modified Lothrop approach for the excision of bilateral frontal sinus tumors. Ear nose and throat journal 2014; 93: 116-9.

5. Anderson P, Sindwani R. Safety and efficacy of the endoscopic modified Lothrop procedure: a systematic review and meta-analysis. Laryngoscope 2009; 119: 1828-33.

6. Becker SS, Bomeli SR, Gross CW, Han JK. Limits of endoscopic visualization and instrumentation in the frontal sinus. Otolaryngol Head Neck Surg 2006; 135: 917-21. 